\title{
Garnet Scintillator-Based Devices for Gamma-Ray Spectroscopy
}

\author{
Owen B. Drury, Nerine J. Cherepy, Thomas A. Hurst, and Stephen A. Payne
}

\begin{abstract}
We are developing scintillator based gamma-ray spectrometers for homeland security and safeguards applications. Improving the energy resolution of such scintillator detectors enables the development of more accurate and effective instruments. We have characterized the response of several $\mathrm{Ce}$ doped garnet scintillators using radioactive sources. These garnet scintillators have multiple decay components. We have acquired pulse height spectra using conventional analog electronics as well as digital electronics with additional postprocessing. By making use of the long decay components, energy resolution can be improved. We are working to implement the inclusion of the long decay times into real-time processing algorithms.
\end{abstract}

\section{INTRODUCTION}

$\mathrm{R}$ ADIATION monitoring, medical imaging, and safeguards applications require efficient gamma-ray spectrometers offering adequate energy resolution to identify weak sources. The effectiveness of these spectrometers is a combination of energy resolution, high $\mathrm{Z}$ and large size. We are developing cerium-doped garnet scintillator-based spectrometers for these applications [1]. Since garnets are oxides, they offer excellent handling and fabrication advantages, compared to the more commonly employed halide scintillators.

The garnet scintillators have multiple decay components where the light output of the longer decays becomes significant. We have found that the maximum Gaussian shaping time of our conventional electronics was not sufficient to determine the shaping time that resulted in the maximum FWHM resolution for these garnets.

In order to more closely obtain the intrinsic resolution, we have acquired pulses digitally for off-line processing. We are developing algorithms that can be implemented real-time to make better use of the long decay components while minimizing the impact on count rate.

\section{PUlse ACQUisition AND ANALYSis}

We present data from two cerium-doped ceramic garnet scintillators; GYGAG (gadolinium yttrium gallium aluminum garnet) fabricated at LLNL, and YAG (yttrium aluminum garnet) from Konoshima Chemical Company. The scintillators were excited using $662 \mathrm{keV}$ gamma rays from a ${ }^{137} \mathrm{Cs}$ source. The scintillators were wrapped with Teflon tape,

Manuscript received November 13, 2009. This work was supported by the Domestic Nuclear Detection Office in the Department of Homeland Security (A. Janos), and performed under the auspices of the U.S. DOE by Lawrence Livermore National Laboratory under Contract DE-AC52-07NA27344.

Owen B. Drury, Nerine J. Cherepy, Thomas A. Hurst, and Stephen A. Payne are with Lawrence Livermore National Laboratory, 7000 East Ave., Livermore, CA 94550, USA (telephone: 925-422-0034, e-mail: drury2@1lnl.gov). and the light was collected with a Hamamatsu R6231U photomultiplier tube (PMT).

The baseline measurements were made using conventional analog electronics. The PMT was readout with a Tennelec TC245 spectroscopy amplifier using $12 \mu \mathrm{s}$ Gaussian shaping. In general, the use of an integrating preamplifier was not required as long as the linearity of the amplifier was carefully monitored. The pulse heights were recorded using an Amptek MCA8000-A. Fig. 1 and Fig. 2 show typical pulse height spectra obtained using the analog electronics.

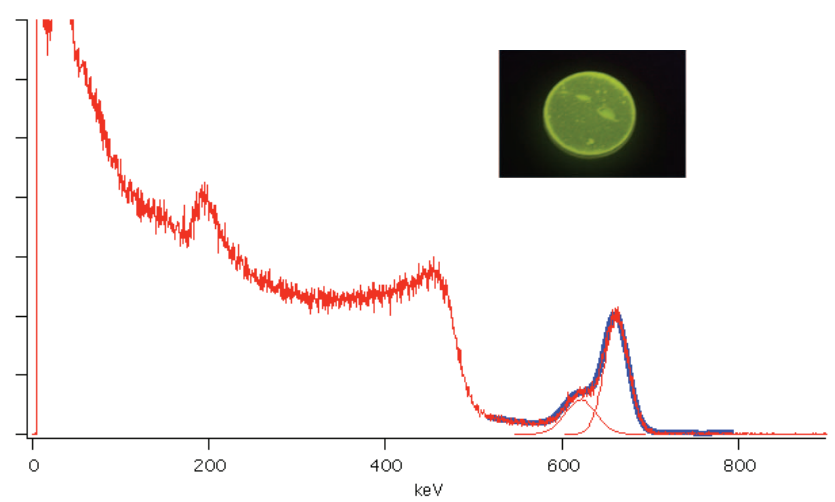

Fig. 1. The pulse height spectrum acquired with analog electronics for the GYGAG(Ce) ceramic (inset) is shown here. The scintillator is $\sim 1 \mathrm{~cm}$ in diameter. This spectrum was acquired using conventional analog spectroscopy electronics. The photo peak has a FWHM resolution of $4.8 \%$ while taking into account the clearly visible Gd X-ray escape peak.

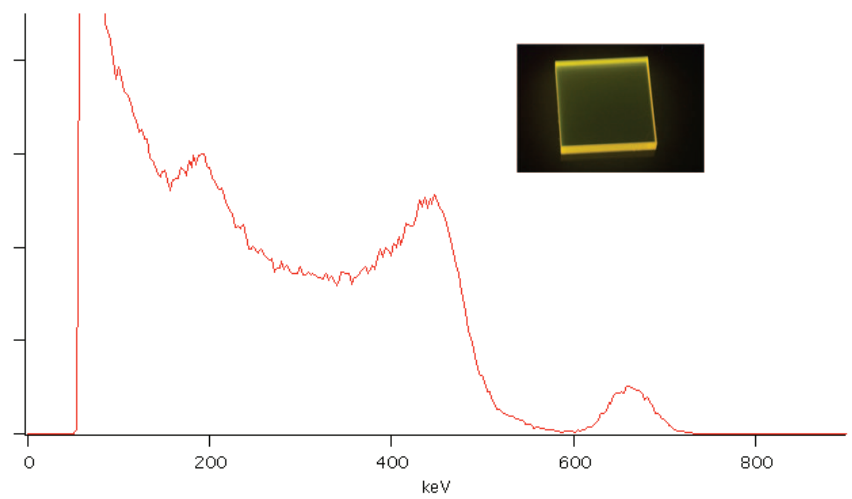

Fig. 2. The analog pulse height spectrum for the $\mathrm{YAG}(\mathrm{Ce})$ ceramic (inset) is shown here. The scintillator is $\sim 1 \mathrm{~cm}$ on a side. This spectrum was also acquired using conventional analog spectroscopy electronics. The photo peak has a FWHM resolution of $8.3 \%$.

For the pulse analysis, the PMT was read out using a selfcontained, USB powered system that includes the high-voltage supply and A/D converter manufactured by Bridgeport Instruments. The pulses were then stored for off-line analysis. 
The garnets contain multiple decay components, visible in Fig. 3 and Fig. 4, that can degrade expected energy resolution performance. The loss of performance is due to their long lifetimes exceeding the shaping time available from the analog spectroscopy amplifier, or exceeding the total number of samples available to a particular data acquisition system. There are competing requirements for the acquisition system to capture data fast enough to maintain the fidelity of the large, prompt features at the beginning of the pulse and to take data long enough to capture the long decay components.

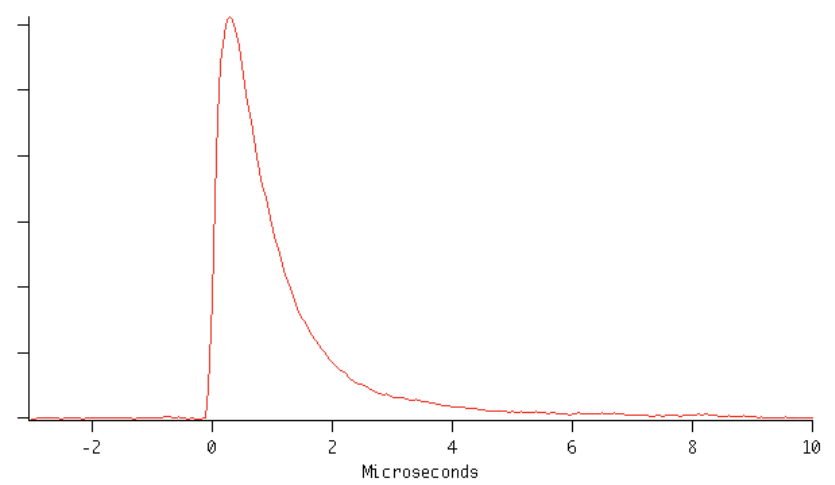

Fig. 3. A typical full energy $662 \mathrm{keV}$ gamma ray absorption scintillation decay trace for $\mathrm{GYGAG}(\mathrm{Ce})$ as acquired by the digital system. Two of the most significant decay times are obvious; and additional very long decay component is present.

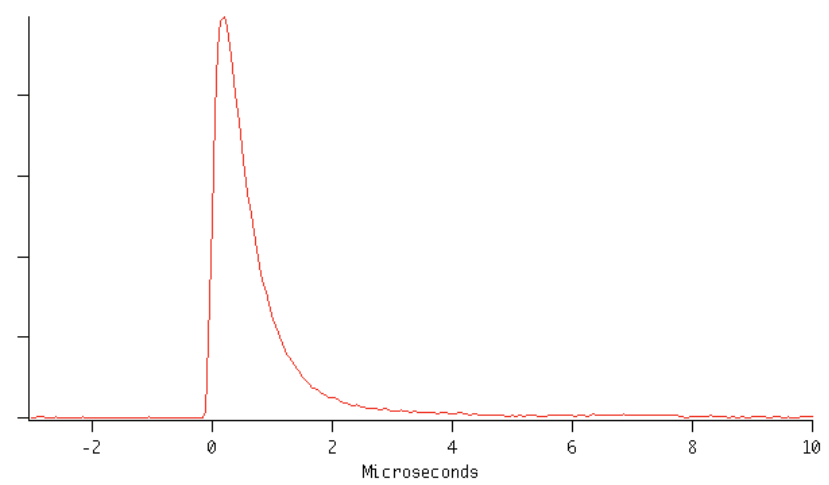

Fig. 4. A typical full energy $662 \mathrm{keV}$ gamma ray absorption scintillation decay trace for $\mathrm{YAG}(\mathrm{Ce})$ as acquired by the digital system.

We begin by fitting the pulses to the exponential function below.

$$
f(t)=\left\{\begin{array}{cc}
a_{0} & t<t_{0} \\
a_{0}+A\left(e^{-\frac{t-t_{0}}{\tau_{d_{1}}}}+\alpha e^{-\frac{t-t_{0}}{\tau_{d_{2}}}}-e^{-\frac{t-t_{0}}{\tau_{r}}}\right) t \geq t_{0}
\end{array}\right.
$$

Where the $\tau$ are the rise and decay times, $a_{0}$ is the pulse offset, $A$ is an overall scaling factor, and $\alpha$ is the scaling of the second decay component relative to the first component. Analytically integrating the pulse above the offset yields

$$
E \propto A\left(\tau_{d 1}+\alpha \tau_{d_{2}}-\tau_{r}\right),
$$

which is proportional to the energy deposited. Fig. 5 shows that the measurement of the energy resolution is improved using this technique from $8.3 \%$ to $7.7 \%$ FWHM for the YAG data set reported here $(\sim 25,000$ points).

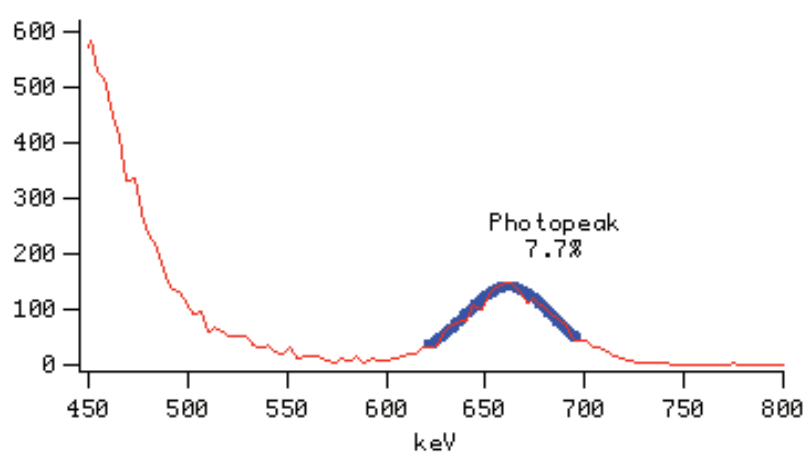

Fig. 5 Expanded view of the photo peak of the energy histogram for the YAG scintillator using pulse analysis. A slight improvement in resolution is obtained compared to the histogram derived from the analog measurements.

The parameters from the fit can also be used to create scatter plots (see Figs. Fig. 7-8) which can be used to select events for inclusion in the final spectrum. To illustrate the point, several selections were made including the main groupings around the rise, and decay times for the data set. The final number of events selected was $\sim 15,000$.

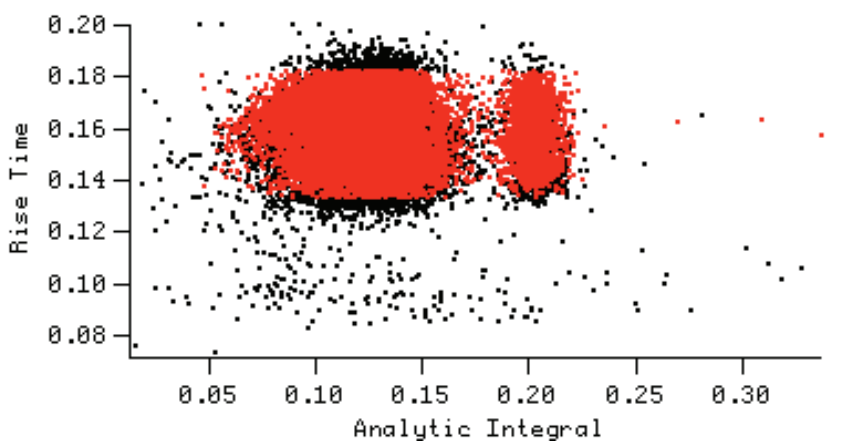

Fig. 6 Scatter plot of the rise time vs the integral for a fit to $\sim 25,000$ pulses acquired from the YAG scintillator. The red points have been "selected" and the black points have not.

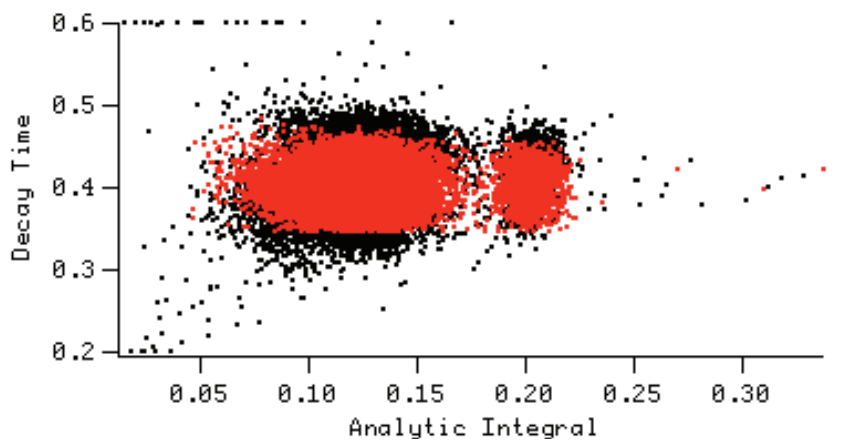

Fig. 7 Scatter plot of the first decay time vs the integral for the YAG data set. 


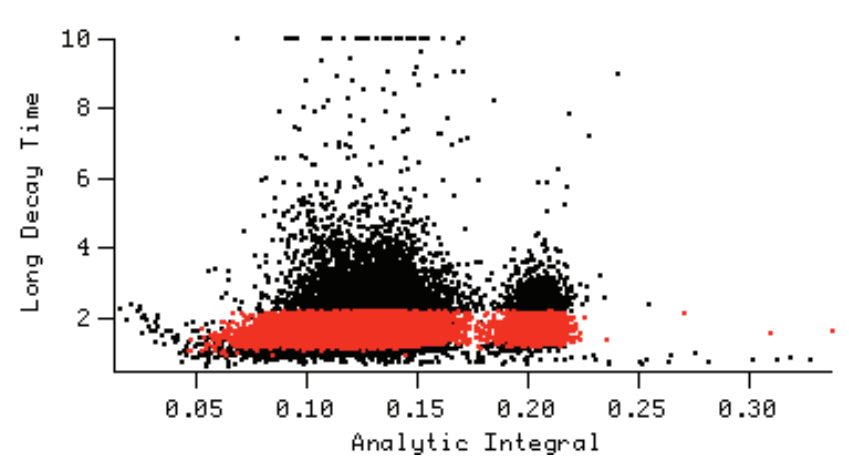

Fig. 8 Scatter plot of the second decay time vs the integral for the YAG data set.

By making the indicated sections, the measured resolution was improved further, to 6.6\% FWHM as shown in Fig. 9. A significant increase in resolution is obtained when compared to either the histogram derived from the analog measurements or the full data set.

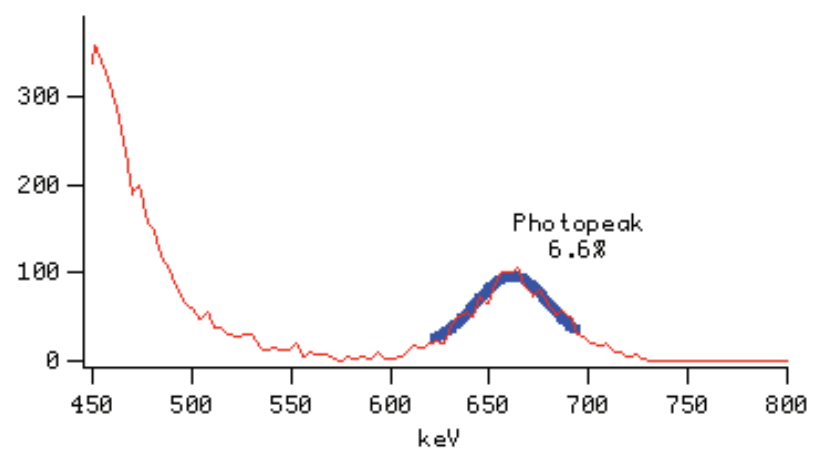

Fig. 9 Expanded view of the photo peak of the energy histogram for the selected subset of data for the YAG scintillator using pulse analysis. Here the resolution has been improved to $6.6 \%$, by discarding $40 \%$ of the events.

\section{CONCLUSIONS}

The garnet scintillators we are developing have very high light yields of $30,000-50,000$ Photons $/ \mathrm{MeV}$, but due to their green-red luminescence spectra and the presence long lifetime decay components, energy resolution results at $662 \mathrm{keV}$ are in the $4.5-7 \%$ range. Conventional pulse acquisition methods are limited by fixed shaping times, while digital acquisition offers the possibility of improved readout based on understanding the pulse shape. Analyzing each event can enable the rejection of anomalous events, which is beneficial to the performance of the spectrometer.

We are analyzing pulses by fitting a representative ideal pulse shape to relevant components. The fitting process can recover some of the information in the tail of the pulse, even if it is truncated. We are then able to calculate the energy deposited during the event.

We have found variations in the amount of improvement of resolution using this method. This variation is an indicator of the overall quality of the scintillator. It can be useful as a diagnostic tool, aiding in the determination of homogeneity.

The key components of this algorithm are being incorporated into an FPGA to be able to obtain similar performance increases real-time, enabling better gamma ray spectrometers.

\section{ACKNOWLEDGMENTS}

We would like to thank J.D. Kuntz, S.E. Fisher, J.J. Roberts, R.D. Sanner, and T.M Tillotson for the fabrication of the GYGAG(Ce) scintillators.

\section{REFERENCES}

[1] N.J. Cherepy, J.D. Kuntz, J.J. Roberts, T.A. Hurst, O.B. Drury, R.D. Sanner, T.M. Tillotson, S.A. Payne, "Transparent ceramic scintillator fabrication, properties and Applications," Proc. of SPIE, vol. 7079, 70790X, 2008. 